\title{
Early prediction of lung lesion progression in COVID-19 patients with extended CT ventilation imaging
}

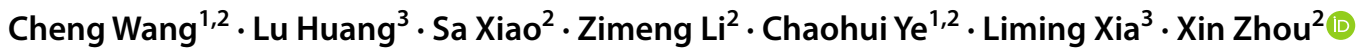

Received: 20 April 2021 / Accepted: 25 May 2021 / Published online: 17 June 2021

(c) The Author(s), under exclusive licence to Springer-Verlag GmbH Germany, part of Springer Nature 2021

\begin{abstract}
Purpose In the prediction of COVID-19 disease progression, a clear illustration and early determination of an area that will be affected by pneumonia remain great challenges. In this study, we aimed to predict and visualize the progression of lung lesions in COVID-19 patients in the early stage of illness by using chest CT.

Methods COVID-19 patients who underwent three chest CT scans in the progressive phase were retrospectively enrolled. An extended CT ventilation imaging (CTVI) method was proposed in this work that was adapted to use two chest CT scans acquired on different days, and then lung ventilation maps were generated. The prediction maps were obtained according to the fractional ventilation values, which were related to pulmonary regional function and tissue property changes. The third CT scan was used to validate whether the prediction maps could be used to distinguish healthy regions and potential lesions. Results A total of 30 patients (mean age \pm SD, $43 \pm 10$ years, 19 females, and 2-12 days between the second and third CT scans) were included in this study. The predicted lesion locations and sizes were almost the same as the true ones visualized in third CT scan. Quantitatively, the predicted lesion volumes and true lesion volumes showed both a good Pearson correlation $\left(\mathrm{R}^{2}=0.80 ; P<0.001\right)$ and good consistency in the Bland-Altman plot (mean bias $=0.04 \mathrm{~cm}^{3}$ ). Regarding the enlargements of the existing lesions, prediction results also exhibited a good Pearson correlation $\left(\mathrm{R}^{2}=0.76 ; P<0.001\right)$ with true lesion enlargements.

Conclusion The present findings demonstrated that the extended CTVI method could accurately predict and visualize the progression of lung lesions in COVID-19 patients in the early stage of illness, which is helpful for physicians to predetermine the severity of COVID-19 pneumonia and make effective treatment plans in advance.
\end{abstract}

Keywords COVID-19 pneumonia $\cdot$ CT ventilation imaging $($ CTVI) $\cdot$ Prediction $\cdot$ Progression

Cheng Wang, Lu Huang, and Sa Xiao contributed equally to this work.

This article is part of the Topical Collection on Technology.

Liming Xia

lmxia@tjh.tjmu.edu.cn

$\triangle$ Xin Zhou

xinzhou@wipm.ac.cn

1 School of Physics, Huazhong University of Science and Technology, Wuhan 430074, China

2 State Key Laboratory of Magnetic Resonance and Atomic and Molecular Physics, National Center for Magnetic

\section{Introduction}

In December 2019, coronavirus disease 2019 (COVID-19) caused by severe acute respiratory syndrome coronavirus 2 (SARS-CoV-2) was first reported in Wuhan, China [1]. By March 2021, more than 118 million people had been infected with COVID-19, and 2.6 million of those infected had died [2]. In this public health emergency, chest computed tomography

Resonance in Wuhan, Wuhan Institute of Physics and Mathematics, Innovation Academy for Precision Measurement Science and Technology, Chinese Academy of Sciences - Wuhan National Laboratory for Optoelectronics, Wuhan 430071, China

3 Department of Radiology, Tongji Hospital, Tongji Medical College, Huazhong University of Science and Technology, Wuhan 430030, China 
(CT) plays an important role in screening and monitoring the progression of COVID-19 pneumonia due to its high efficiency [3]. The typical CT features of COVID-19 pneumonia include ground glass opacities (GGOs), GGOs with consolidation, eccentric paving patterns, and consolidation [4]. In accordance with the guidelines of the diagnosis and treatment of COVID19 pneumonia, patients are suggested to undergo more than one chest CT scan during hospitalization [5].

During the treatment of COVID-19 patients, it is advantageous to identify patients who are likely to progress to a severe or critical stage as soon as possible [6]. Previous studies have shown that CT structural features, combined with clinical and laboratory biomarkers, are a promising tool to predict the clinical progression of COVID-19 [7-13]. However, previous methods focused on the prediction of the overall progression of the disease, and it is difficult to determine the spatial location and the size of the upcoming lung lesions (e.g., the new GGO and/or consolidation) in COVID-19 patients. Therefore, it is necessary to develop a prediction method to help physicians accurately assess COVID-19 disease progression with a clear illustration that allows for early determination of the area that will be affected by COVID-19 pneumonia [14].

Generally, there is an incubation period of several days before the emergence of lung lesions on CT imaging in COVID-19 patients $[15,16]$. During this incubation period, regional lung function might have been impaired; this functional impairment can be detected by lung function imaging [17-19]. Preliminary studies have used single-photon emission computed tomography [20] to discover regional lung gas-exchange impairments (e.g., ventilation defects) in COVID-19 patients in the early stage of the disease [21, 22]. Hyperpolarized noble gas (such as ${ }^{3} \mathrm{He}$ or ${ }^{129} \mathrm{Xe}$ ) magnetic resonance imaging (MRI) [23] has also been used for the anatomical structure and functional imaging of the human lung, and recently, our group detected damaged lung gas-exchange function with hyperpolarized ${ }^{129} \mathrm{Xe}$ MRI in discharged COVID-19 patients [24]. In addition, CT ventilation imaging (CTVI) using two chest CT scans is able to detect regional lung function [25]. The CTVI method generates the ventilation images from a pair of inspiration and expiration CT images [26-28]. The regional volume changes due to ventilation are calculated based on CT regional values, and the regions of greater changes in CT values correspond to higher relative ventilation. Considering that many COVID-19 patients have undergone more than one chest CT scan, CTVI has a good foundation in the lung function impairment research on COVID-19.

Based on the above, we intend to use CTVI to predict the progression of lung lesions in COVID-19 patients in the early stage of the disease. Nevertheless, the conventional CTVI method uses two CT scans acquired on the same day [25-28], while the CT scans of the COVID-19 patients are usually acquired on different days. Thus, we propose an extended
CTVI method for two CT scans acquired on different days. Then, the prediction maps generated from the proposed method are used to distinguish the healthy lung regions and potential impaired lung regions. This method could predict the progression of lung lesions accurately, help physicians predetermine the severity of COVID-19 pneumonia, and assist them in making effective treatment plans in advance.

\section{Materials and methods}

\section{Study subjects}

From January 13 to March 28, 2020, COVID-19 patients who were confirmed by using real-time reverse-transcriptase polymerase chain reaction were retrospectively enrolled from Tongji Hospital, Wuhan, China. The inclusion criteria of the patients were as follows: (1) each patient underwent three chest CT scans [the first two CT scans (referred to as CT1 and CT2) were used for prediction, and the third CT scan (referred as CT3) was used to validate the predicted results], (2) the CT scans of the patients were acquired in the progressive phase of the disease, and (3) the patients had no previous history of pulmonary disease. The local ethical review board approved this retrospective study and waived the requirement to obtain individual informed consent.

\section{Extended CT ventilation imaging}

In the acquisition process, all chest CT scans were performed in the supine position. The $\mathrm{CT}$ acquisition parameters were as follows: tube voltage $=100-120 \mathrm{kVp}$, matrix $=512 \times 512$, slice thickness $=1-1.25 \mathrm{~mm}$, field of view $=350 \mathrm{~mm} \times 350 \mathrm{~mm}$, and spiral pitch factor $=0.81-1.375$.

In CT image preprocessing, lung masks of CT1 and CT2 images were initially segmented with the seed region growth algorithm provided in 3D Slicer software (version 4.2) [29] and then manually corrected by two radiologists (with more than 10 years of experience in pulmonary imaging diagnosis). Then, CT1 and CT2 images were spatially coregistered using the B-spline deformation registration algorithm provided in Elastix software (version 4.9) [30]. After that, CT1 and CT2 images were smoothed by the median filter. Finally, the nonaerated lung regions (e.g., vasculature) and the visible lesions were marked and excluded from the CTVI analysis [31].

After image preprocessing, the lung ventilation maps were calculated according to CTVI theory. In this theory, chest CT scans measure the density [Hounsfield units (HUs)]] of the pulmonary voxel, and air ventilation causes a change in the $\mathrm{HU}$ value [25]. The conventional CTVI method used two CT scans with different respiratory phases that were acquired on the same day (e.g., the end-inspiration and end-expiration of a 4D CT scan) to generate a lung ventilation map [25-28]. However, 
during the clinical hospitalization of COVID-19 patients, the CT1 and CT2 scans of the patients were usually acquired on different days. Thus, in this paper, we adapted the CTVI method to evaluate two lung CT scans acquired on different days.

Herein, we derived the mathematical relationship between ventilation and $\mathrm{HU}$ values to generate a lung ventilation map. A pulmonary voxel could be considered a combination of air content and tissue content [32]. The tissue includes cells, blood, collagen, water, etc., which have approximately the same density of water. The air volume $\left(\mathrm{V}_{\text {air }}\right)$ and tissue volume $\left(\mathrm{V}_{\text {tissue }}\right)$ of that voxel could be calculated based on the total volume $\left(\mathrm{V}_{\text {total }}\right)$ and total $\mathrm{HU}$ value $\left(\mathrm{HU}_{\text {total }}\right)$ of that voxel (see Appendix). Then, for that pulmonary voxel in $\mathrm{CT} 1$ and CT2 images, the corresponding air volume $\left(\mathrm{V}_{\text {air1 }}\right.$ and $\left.\mathrm{V}_{\text {air2 }}\right)$ and tissue volume $\left(\mathrm{V}_{\text {tissue } 1}\right.$ and $\left.\mathrm{V}_{\text {tissue } 2}\right)$ are

$V_{\text {air } 1}=\frac{H U_{1}}{-1000} * V_{1}, V_{\text {tissue } 1}=\left(1-\frac{H U_{1}}{-1000}\right) * V_{1}$

$V_{\text {air } 2}=\frac{H U_{2}}{-1000} * V_{2}, V_{\text {tissue } 2}=\left(1-\frac{H U_{2}}{-1000}\right) * V_{2}$

where $\mathrm{V}_{1}$ and $\mathrm{HU}_{1}$ and where $\mathrm{V}_{2}$ and $\mathrm{HU}_{2}$ represent the total volume and total $\mathrm{HU}$ value of that voxel in the $\mathrm{CT} 1$ and $\mathrm{CT} 2$ scans, respectively.

Then, the fractional ventilation (FV) value between the CT1 and CT2 scans of the pulmonary voxel is defined as the breathing-induced air volume change [26-28] and could be calculated as

$F V=\left\{\begin{array}{l}\left(V_{\text {air } 1}-V_{\text {air } 2}\right) / V_{a i r 2}, i f V_{a i r 1}>V_{\text {air } 2} \\ \left(V_{\text {air } 2}-V_{\text {air } 1}\right) / V_{\text {air } 1}, \text { if } V_{\text {air } 2}>V_{\text {air } 1}\end{array}\right.$

Assuming the tissue volume of that pulmonary voxel has little difference between CT1 and CT2,

$V_{\text {tissue } 1}=V_{\text {tissue } 2}$

Then, the FV of that pulmonary voxel between CT1 and CT2 is

$F V=\left\{\begin{array}{l}\frac{-1000}{\left(1000+H U_{1}\right) H U_{2}} *\left(H U_{2}-H U_{1}\right), i f V_{a i r 1}>V_{a i r 2} \\ \frac{-1000}{\left(1000+H U_{2}\right) H U_{1}} *\left(H U_{1}-H U_{2}\right), i f V_{a i r 2}>V_{a i r 1}\end{array}\right.$

Based on Eq. (4), the lung ventilation map could be obtained with the calculated FV values. The validation of the accuracy of FV values was shown in Fig. S1 of the Supplementary material.

\section{Prediction of lung lesion progression}

The concept of predicting the progression of lung lesions in COVID-19 patients is shown in Fig. 1 and mainly includes four steps. In the first step, CT1 (Fig. 1a) and CT2 (Fig. 1b) images were used to generate the lung ventilation map (Fig. 1c) with the extended CTVI method. In the second step, the pulmonary voxels in the lung ventilation map were divided into three categories (P1, P2, and P3) according to the FV value of each pulmonary voxel, as shown in Fig. 1d. In the third step, the three categories were displayed as different colors and visualized on a prediction map, as shown in Fig. 1e. The blue, black, and yellow color voxels represent the $\mathrm{P} 1, \mathrm{P} 2$, and $\mathrm{P} 3$ voxels, respectively, in the prediction map. P1 was predicted to be a healthy region, and P2 and P3 were predicted to be lesions. Finally, a follow-up CT3 scan (Fig. 1f) was used to validate the predicted results.

The second step was critical for predicting the progression of lung lesions in COVID-19 patients. The pulmonary voxels were divided into three categories in this step. In the first category, the tissue volume of the voxel had little difference between CT1 and CT2 scans, and the voxel had normal ventilation function. Therefore, the voxel had a normal FV value (referred to as the P1 voxel). In the second category, the tissue volume of the voxel had little difference between CT1 and CT2 scans, but the voxel had impaired ventilation function. Accordingly, the voxel should have an abnormally lower FV value (referred to as the P2 voxel). In the third category, the tissue volume of the voxel had some differences between CT1 and CT2 scans, which indicated the trends of lesions and the sharp increase of HU values. Thus, the voxel should have an abnormally higher FV value (referred to as the $\mathrm{P} 3$ voxel) due to the large value of $\left(\mathrm{HU}_{2}-\mathrm{HU}_{1}\right)$ in Eq. (4).

Specifically, we used a threshold method to distinguish $\mathrm{P} 1, \mathrm{P} 2$, and $\mathrm{P} 3$ voxels. The voxels with FV values below $A$ were categorized as $\mathrm{P} 2$, the voxels with $\mathrm{FV}$ values above $B$ were categorized as $\mathrm{P} 3$, and the voxels with $\mathrm{FV}$ values between $A$ and $B$ were categorized as P1. In this work, we empirically set the threshold $A=0.01$ and threshold $B=1.0$.

\section{Statistical analysis}

The lesions on the maximum lesion slice of the CT2 scan and the follow-up CT3 scan were manually segmented and corrected by the two radiologists with more than 10 years of experience in pulmonary imaging diagnosis. Continuous variables are expressed as the means \pm standard deviations (SDs), and categorized variables are expressed as frequencies and percentages. The lesion volume in the CT3 scan was used as the true lesion volume. Paired 2-tailed Student's $t$-test was used to evaluate the differences between the lesion volumes in prediction maps and follow-up CT3 scans. The Pearson correlation and Bland-Altman plot were used to evaluate the agreement between the predicted lesion volumes and the true lesion volumes. The Pearson correlation between the predicted lesion enlargements and the true lesion enlargements was analyzed. The relation between the relative prediction error and the time interval of CT3-CT2 was plotted. All statistical analyses were performed by using SPSS statistical software (version 20.0), and $P<0.05$ was considered statistically significant. 

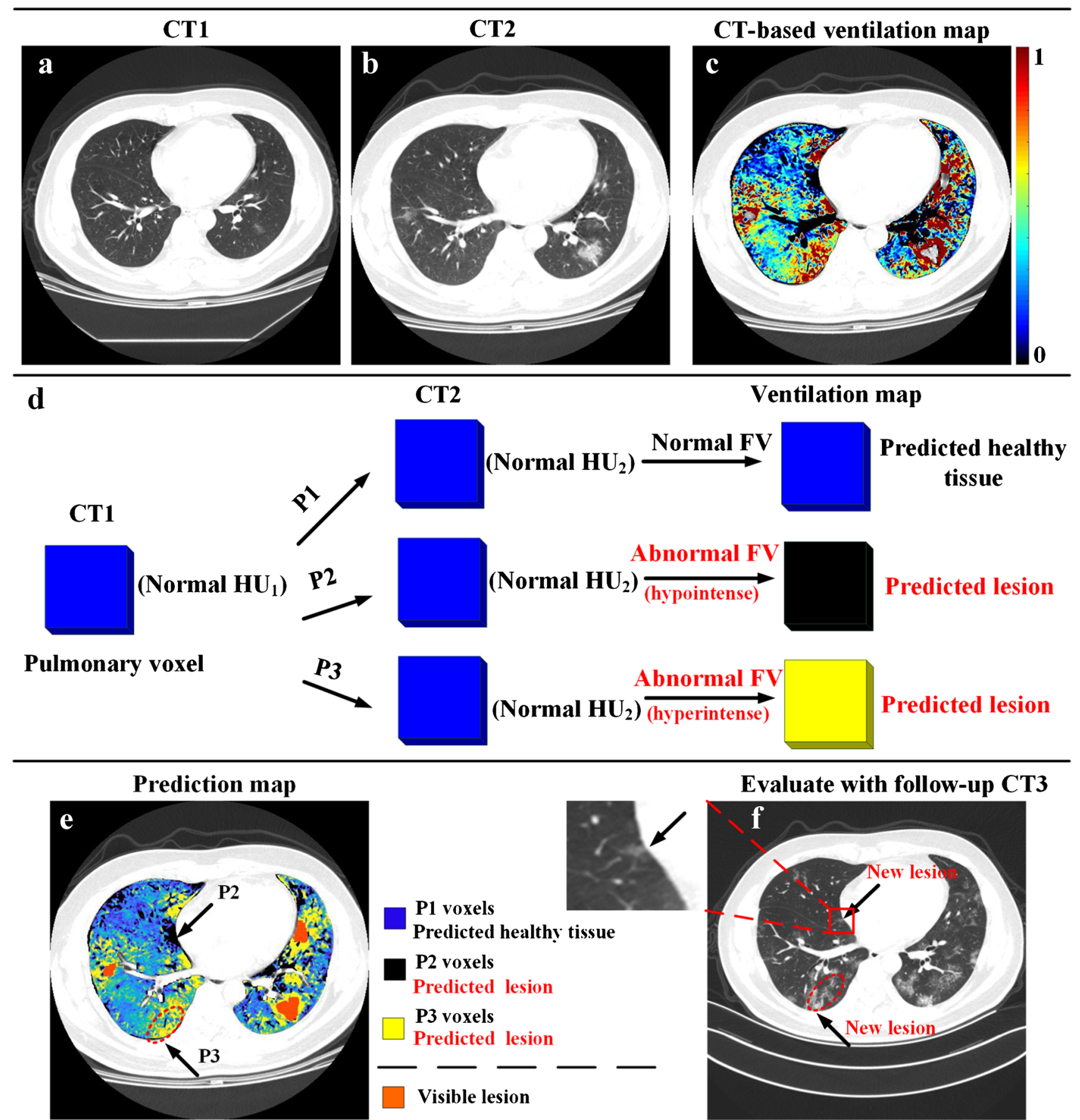

P1 voxels

Predicted healthy tissue

P2 voxels

Predicted lesion

P3 voxels

Predicted lesion

Visible lesion

Evaluate with follow-up CT3

Fig. 1 The concept of predicting the progression of lung lesions in COVID-19 patients. First, the a CT1 and b CT2 images were used to generate the $\mathbf{c}$ lung ventilation map by the extended CTVI method. Then, the d pulmonary voxels were categorized into three types (P1, $\mathrm{P} 2$, and $\mathrm{P} 3$ ) according to the FV values of the CT-based ventilation

\section{Results}

\section{Participant demographic}

A total of 30 COVID-19 patients (mean age \pm SD, $43 \pm 10$ years, and 19 females) were included in this study. map and were used to create the e prediction map. P1 was predicted to be a healthy region, and $\mathrm{P} 2$ and $\mathrm{P} 3$ were predicted to be lesions. Finally, the predicted results were validated by the follow-up CT3 scan. The visible lesions on the CT2 image were marked in orange in the prediction map and excluded from the prediction

The most common symptom of the patients at symptom onset was fever $(92.0 \%)$. During the observation period, leukocytes were one of the main abnormalities observed in laboratory inspection. The mean $\pm \mathrm{SD}$ of the time intervals between symptom onset and CT1 scans, between CT1 scans and CT2 scans, and between CT2 scans and CT3 
scans of the patients were $1.6 \pm 1.9$ days, $4.0 \pm 2.1$ days, and $5.8 \pm 2.6$ days, respectively. The mean \pm SD of the relative change in lung volumes between the CT1 and CT2 scans of the patients was $11.3 \% \pm 9.9 \%$. The detailed demography and clinical characteristics of the patients are shown in Table 1.

\section{Prediction results}

Figure 2 displays the CT1 scan, CT2 scan, CT3 scan, and prediction maps of three representative COVID-19 patients. For the first patient (female, 39 years old), CT1 and CT2 scans showed no positive findings, and the prediction map was mainly composed of $\mathrm{P} 1$ voxels (blue color), which predicted that the lungs of that patient were healthy. As verification, the follow-up CT3 scan showed negative findings. For the second patient (male, 30 years old), CT1 and CT2 scans showed negative findings, but the prediction map had a large number of $\mathrm{P} 2$ voxels (black color) in the right lower lobe. This predicted that the right lower lobe would develop into regions with lesions. Then, visible lesions appeared in that lobe in the CT3 results, which verified the prediction result. For the third patient (male, 40 years old), CT1 showed slight lesions in the right lung, and the CT2 scan showed visible lesions in the lower lobes of both lungs. The corresponding prediction map had a large number of P3 voxels (yellow color) in other regions of both lungs, although these voxels were normal on CT1 and CT2 scans. These P3 voxels predicted that the corresponding regions would develop visible lesions. As expected, the CT3 scan showed that all P3 voxels became visible lesions.
After illustrating the representative prediction results, we quantitatively analyzed the accuracy of the predicted results. Table 2 shows the lesion volumes in the maximum lesion slice of the CT2 scan, the prediction map, and the follow-up CT3 scan of the patients. Additionally, there was no significant difference between the prediction results and the true results $(P=0.865)$, as shown in Fig. 3a. The Pearson correlation analysis between the predicted lesion volumes and the true lesion volumes of all patients is displayed in Fig. 3b. The predicted volumes and the true volumes had a good correlation $\left(\mathrm{R}^{2}=0.80 ; P<0.001\right)$. Figure $3 \mathrm{c}$ shows the Bland-Altman plot of these 2 measurements (the prediction and the follow-up). The mean bias \pm SD was $0.04 \pm 1.23 \mathrm{~cm}^{3}$ ( $95 \%$ limit of agreement, $-2.37 \mathrm{~cm}^{3}$ to $2.45 \mathrm{~cm}^{3}$ ). The above results indicated that the predicted lesion volumes and the true lesion volumes were in good agreement. In addition, the predicted lesion enlargements and the true lesion enlargements of all patients are also shown in Table 2. There was no significant difference between the prediction results and the true results $(P=0.884)$. Figure $3 \mathrm{~d}$ shows the Pearson correlation analysis between the predicted lesion enlargements and the true lesion enlargements of all patients. The predicted enlargements and the true enlargements had a good correlation $\left(\mathrm{R}^{2}=0.76 ; P<0.001\right)$.

In addition, the performance of the proposed prediction maps in negative CT was evaluated. Figure 4 shows the CT1 scan, CT2 scan, CT3 scan, and the prediction maps of three representative COVID-19 patients without positive lesions in both CT1 and CT2 scans. For the first patient (female, 43 years old), the prediction map showed that some regional lesions (indicated by red cycles) would appear in the left upper lobe. For the second patient (female, 60 years old),
Table 1 The demographic and clinical characteristics of the COVID-19 patients

\begin{tabular}{ll}
\hline Patient characteristics & Patients $(n=30)$ \\
\hline Age, years & $42.7 \pm 10.0(29-72)$ \\
Sex, female & $19(63.3 \%)$ \\
Initial symptoms & \\
Fever $^{\mathrm{a}}$ & $23(92.0 \%)$ \\
Cough $^{\mathrm{a}}$ & $12(48.0 \%)$ \\
Fatigue $^{\mathrm{a}}$ & $9(36.0 \%)$ \\
Myalgia $^{\mathrm{a}}$ & $6(24.0 \%)$ \\
Laboratory studies within the first CT scan $^{\text {Leukocytes }}{ }^{\mathrm{a}}, 10^{9} / \mathrm{L}$ (normal, 1.1-3.2) & \\
Lactate dehydrogenase $^{\mathrm{a}}, \mathrm{U} / \mathrm{L}$ (normal, 120-250) & $7.8 \pm 4.7(2.9-20.8)$ \\
Time course of chest CT scans & $253 \pm 119(144-733)$ \\
Time interval between onset and CT1 ${ }^{\mathrm{a}}$, days & \\
Time interval between CT1 and CT2, days & $1.6 \pm 1.9(0-6)$ \\
Time interval between CT2 and CT3, days & $4.0 \pm 2.1(2-11)$ \\
Relative change of lung volumes between CT1 and CT2 & $5.8 \pm 2.6(2-12)$ \\
\hline
\end{tabular}

Values are presented as the mean $\pm \mathrm{SD}$ (range) or $n(\%)$.

${ }^{a}$ Data were missing for five patients. 


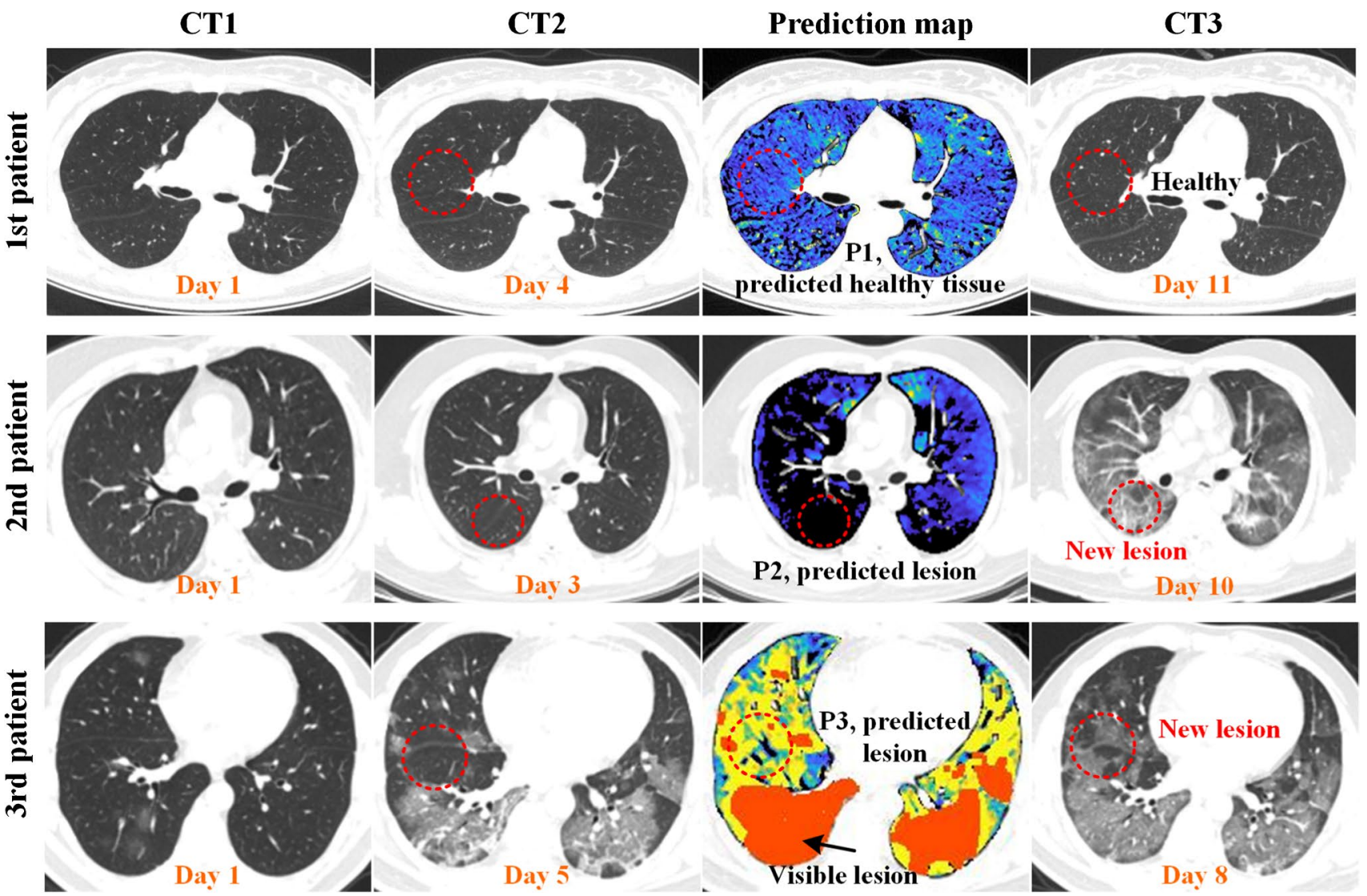

Fig. 2 CT1 scan, CT2 scan, CT3 scan, and the prediction maps of three representative COVID-19 patients. The prediction map of the first patient (female, 39 years old) mainly contained P1 voxels (blue color). The prediction map of the second patient (male, 30 years old) had a large number of P2 voxels (black color). The prediction map of the third patient (male, 40 years old) had a large number of P3 voxels (yellow color). The $\mathrm{P} 1$ voxels predicted the healthy lung regions. The $\mathrm{P} 2$ and P3 voxels predicted potential lung lesions. These prediction results were verified in the follow-up CT3 scan

the regions indicated by red circles. For the second patient (male, 57 years old), the prediction map demonstrated that the lesions on both lungs would enlarge significantly in the regions indicated with a yellow color. For the third patient (male, 40 years old), the prediction map revealed that the lesions on both lower lobes were enlarged in the regions indicated with a yellow color. Then, the enlargements of these lesions were verified by a follow-up CT3 scan. To further evaluate the regional function and tissue property changes of the existed lesions, the FV values and corresponding prediction map of visible lesions were calculated, which were shown in Fig. S2 of the Supplementary material.

To evaluate the relation between the accuracy of the prediction maps and the time interval of CT3-CT2 scans,
Table 2 The lesion volumes and enlargements (mean $\pm \mathrm{SD})$ in the maximum lesion slice of the COVID-19 patients

\begin{tabular}{lllll}
\hline & CT2 & Follow-up CT3 & Prediction & $P$ value \\
\hline Lesion volume $\left(\mathrm{cm}^{3}\right)$ & $1.09 \pm 0.91$ & $2.22 \pm 2.59$ & $2.26 \pm 1.89$ & 0.865 \\
Lesion enlargement $\left(\mathrm{cm}^{3}\right)$ & N/A & $1.13 \pm 2.31$ & $1.17 \pm 1.55$ & 0.884 \\
\hline
\end{tabular}



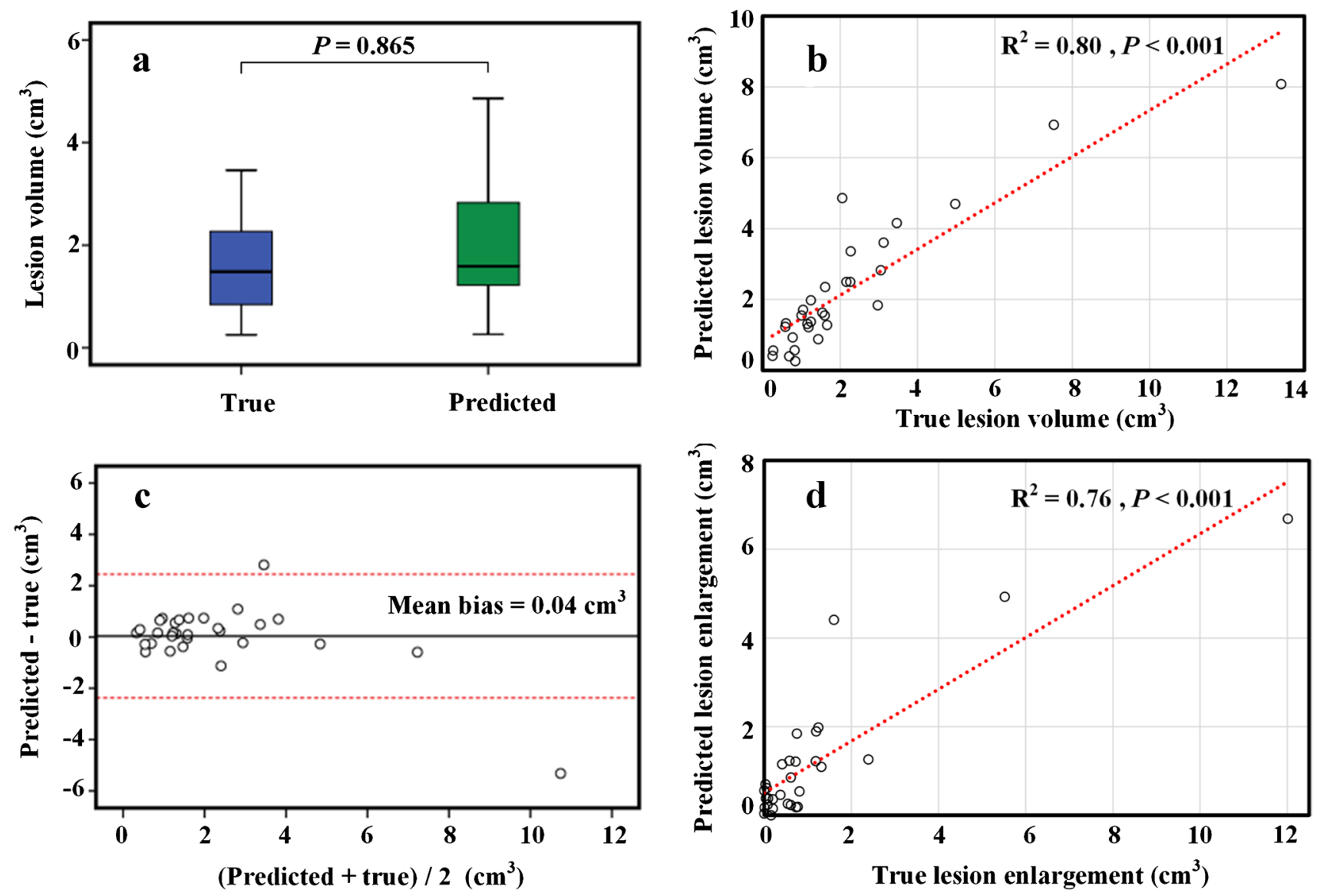

Fig. 3 Quantitative analysis of the accuracy of the predicted results. a The group results of true lesion volumes and the predicted lesion volumes in the maximum lesion slice of the COVID-19 patients. b The correlation analysis results between the predicted lesion volumes and

statistical analysis was performed on the relative errors and the time intervals. Figure 6a displays the relation between the relative errors of the lesion volumes and the time intervals. The mean \pm SD of relative errors between the predicted lesion volumes and the true lesion volumes for the patients was $0.37 \pm 0.41$. The relative errors were relatively lower in the middle of the time intervals (5-8 days). Moreover, the mean relative errors for the time intervals of 2-4 days, 5-8 days, and $9-12$ days were $0.46,0.28$, and 0.36 , respectively. The interval of 5-8 days had a lower relative prediction error regarding the lesion volume. Figure $6 \mathrm{~b}$ displays the relation between the relative errors of the lesion enlargements and the time intervals. The mean $\pm \mathrm{SD}$ of relative errors between the predicted lesion enlargements and the true lesion enlargements for the patients was $0.87 \pm 0.79$. The relative errors were relatively lower in the middle time interval (5-8 days). Moreover, the mean relative errors for the time intervals of 2-4 days, 5-8 days, and 9-12 days were 1.08, 0.62, and 0.92, respectively. The interval of 5-8 days had a lower relative prediction error regarding lesion enlargement. the true lesion volumes. c Bland-Altman plot of the predicted lesion volumes and the true lesion volumes. $\mathbf{d}$ The correlation analysis results between the predicted lesion enlargements and the true lesion enlargements

\section{Discussion}

Chest CT plays an important role in screening and monitoring the progression of COVID-19 pneumonia, and there is an incubation period of several days before the emergence of lung lesions in CT scans $[15,16]$. Generally, lung function impairments can be detected earlier than structural lesions on CT scans [22, 24]. Therefore, an extended CTVI method was proposed in this study to detect potential functional and tissue property changes in COVID-19 patients. The proposed method could predict the progression of lung lesions in the early stage of COVID-19 patients. Furthermore, through the proposed prediction maps, both the spatial locations and sizes of the lesions could be predicted.

The performance of the proposed method was validated by follow-up CT scans. The spatial locations and sizes of lesions in the predicted results had good agreement with the true lesions. For the sizes of the lesions, all the statistical results, such as those of the $t$-test, Pearson correlation, and Bland-Altman plot, indicated good predictive performance. 


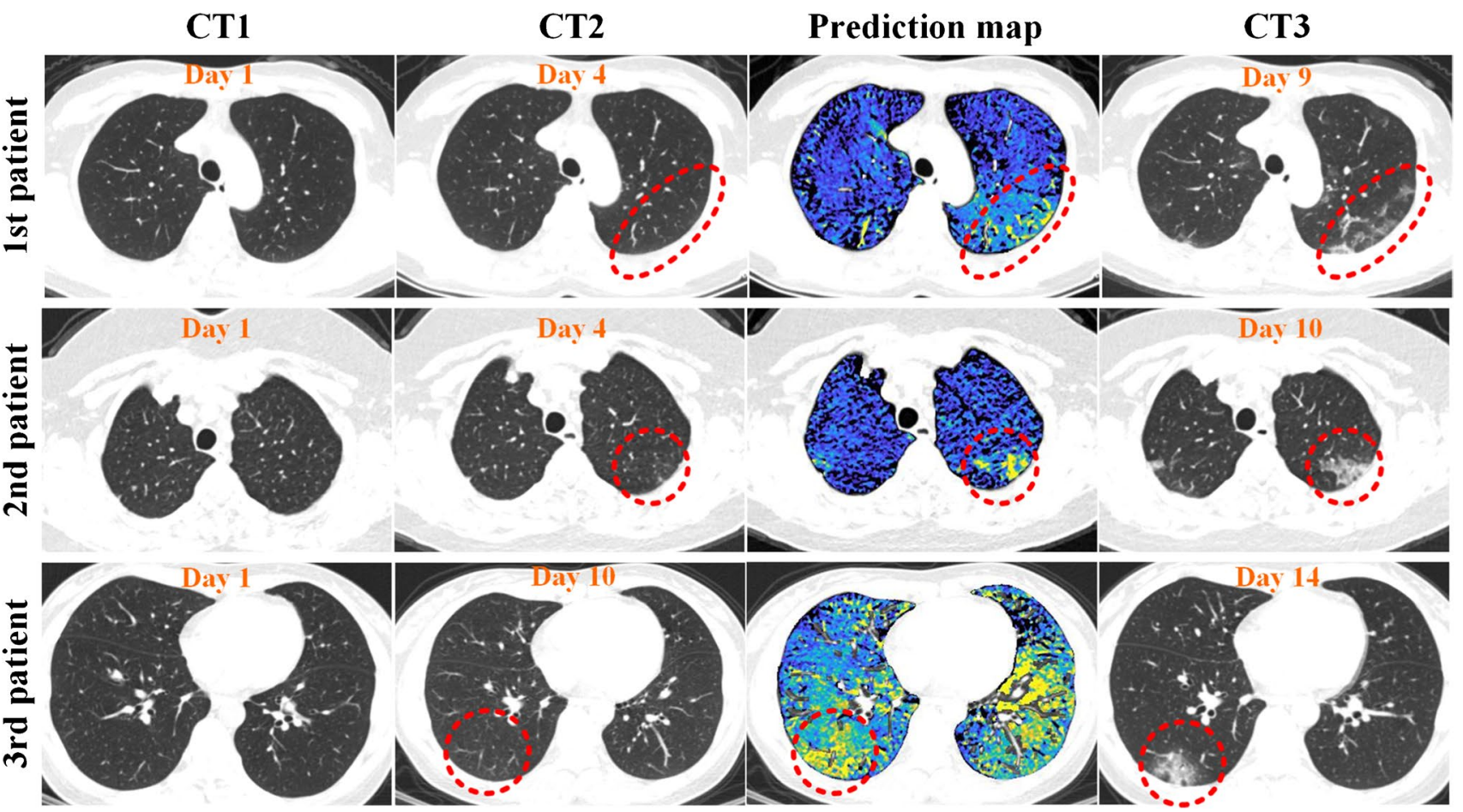

Fig. 4 CT1 scan, CT2 scan, CT3 scan, and the prediction maps of three representative COVID-19 patients. In CT1 and CT2 scans of the first patient (female, 43 years old), the second patient (female, 60 years old), and the third patient (female, 36 years old), no lesion was discovered. The prediction maps predicted that some regions (indicated by red cycles) in these patients would have lesions. The follow-up CT3 scan confirmed these predictions
For the locations of the lesions, there was good agreement between the true results and the predicted regions indicated by red circles in Figs. 2, 4, and 5. All of these results indicated that the proposed method had good performance in the early prediction of lung lesion progression in COVID-19 patients. Note that all the prediction maps of the patients could be obtained several days (mean $=5.8$ days) earlier than the follow-up CT scan. This could help physicians predetermine the severity of COVID-19 pneumonia earlier and make corresponding treatment plans in advance.

Different from the conventional CTVI method [26-28], we proposed an extended CTVI method adapted to use two lung CT scans acquired on different days in this work. The mathematical relationship between the fractional ventilation values and the $\mathrm{HU}$ values was derived considering the different lung volumes of CT1 and CT2 scans. In addition, the effect of tissue change was considered in the generation of the prediction map, and the abnormally higher FV values were attributed to this effect. In the generation of the prediction map, thresholds $A$ and $B$ were used to distinguish the healthy regions and lesions. However, few studies using the CTVI method have studied these thresholds [33, 34]. Therefore, we referenced studies that used hyperpolarized gas MRI [34]. Ten percent of the mean ventilation value of the population could be used as a threshold to detect ventilation defects [35]. In this work, the mean ventilation value (i.e., the mean value of the relative change in lung volumes) of the patients was $11.3 \%$. In addition, the FV values were usually within $1.0[26,27]$. Thus, we empirically set $A=0.01$ and $B=1.0$. The predicted results confirmed the suitability of the threshold values. In the future, the optimization method for $A$ and $B$ could be explored.

Moreover, the time interval of 5-8 days between the CT2 scan and the follow-up CT3 scan was shown to have the best performance for predicting lung lesions. The time intervals could cause the prediction map to not be entirely consistent with the follow-up CT3. If the time interval was too short, some regional lesions indicated by the prediction map might need more time to develop as visible lesions on CT3 scans. For example, in the third patient of Fig. 4, some yellow regions in the left lung of the prediction map did not have lesions in the CT3 scan, which may be due to the insufficient progression time between CT2 and CT3 scans (4 days). If the time interval was too long, some lesions indicated by the prediction map might have been absorbed, and some lesions that were not reflected in the prediction map might appear. For example, the left lower lung lesions in Fig. If were not entirely consistent with the prediction, which may be due to the long progression time between CT2 and CT3 scans (12 days). These discrepancies could generate some differences between 

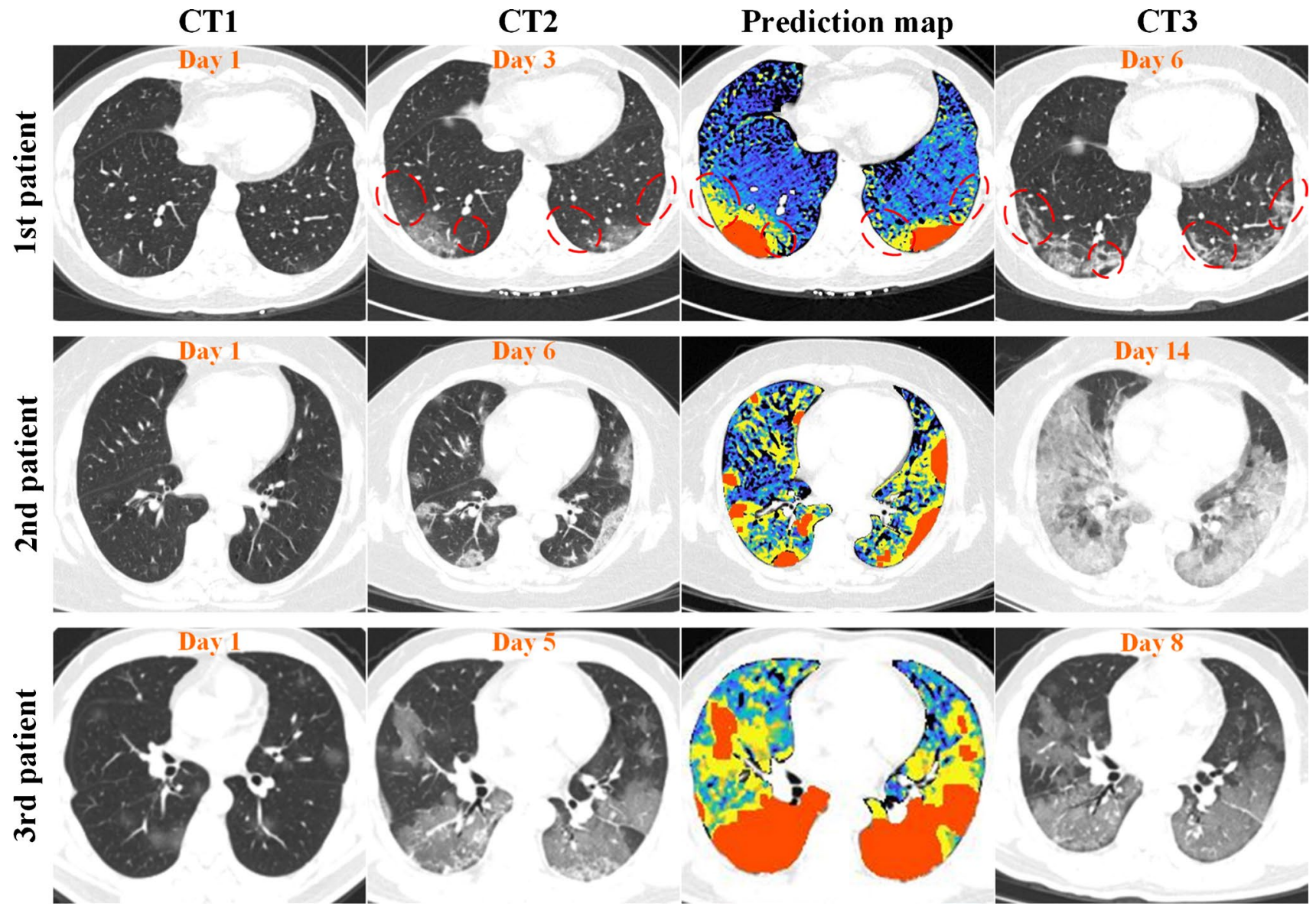

Fig. 5 CT1 scan, CT2 scan, CT3 scan, and the prediction maps of three representative COVID-19 patients. In CT2 scans of the first patient (female, 60 years old), the second patient (male, 57 years old), and the third patient (male, 40 years old), some lesions were discovered (marked with an orange color in the prediction maps).
The prediction maps showed that the lesions in these patients would enlarge in the regions indicated by red circles and a yellow color. The enlargements of these lesions were verified by the follow-up CT3 scan
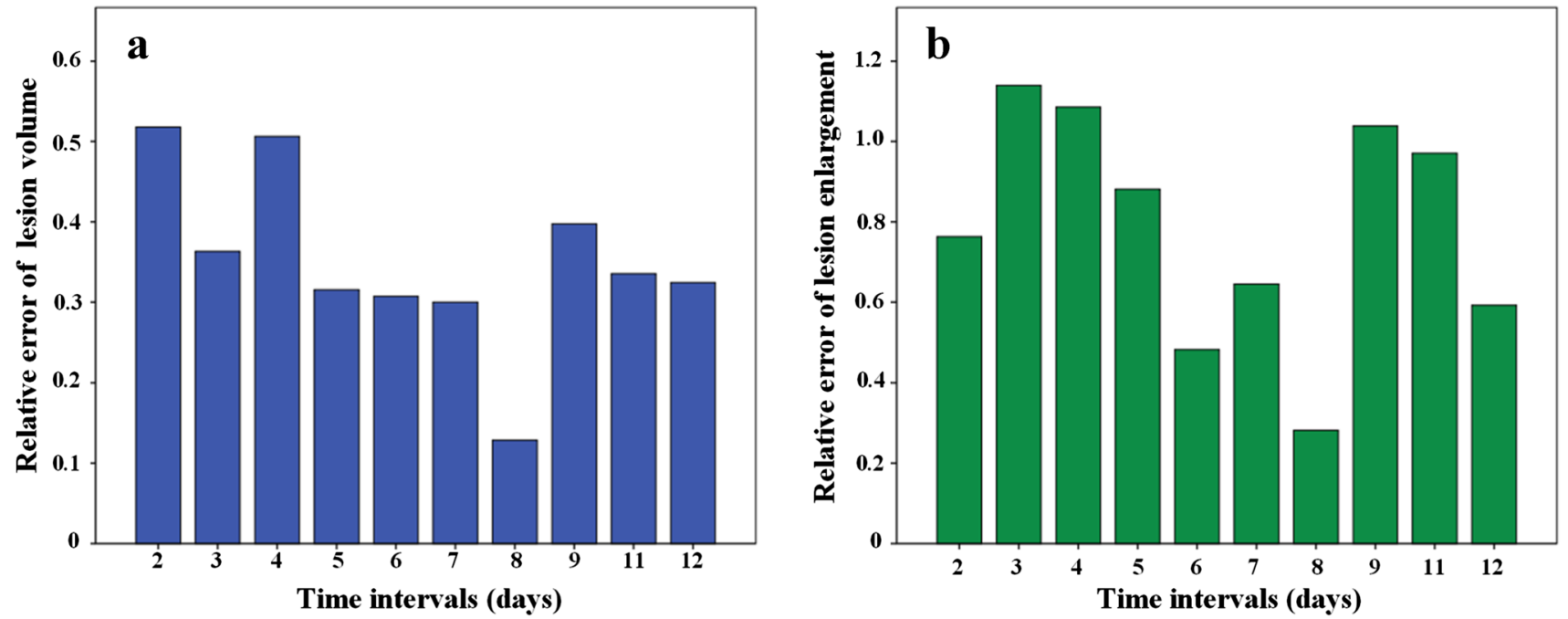

Fig. 6 The relation between the time intervals of CT3-CT2 scans and a the relative errors of the lesion volumes and the relation between the time intervals of CT3-CT2 scans and b the relative errors of the lesion enlargements 
the predicted lesion volumes and the follow-up CT3 lesion volumes and cause outliers in the quantitative analysis, as shown in Fig. 3. In this work, the mean time interval between onset and the CT2 scan was approximately 6 days. Thus, the best time interval between onset and CT3 was 11-14 days, which was consistent with the peak (approximately 2 weeks) of COVID-19 disease symptoms [36].

The information that 5-8 days between the CT2 and followup CT3 scans had the best performance for predicting lung lesions could provide several benefits for the treatments of COVID-19 patients in clinical. First, it could provide an early warning and relatively sufficient time for physicians to make intervention treatments in advance to avoid the incidence of the severe ill for some patients. Second, it could prevent overtreatment for some patients without severe progress in this period and reduce the medical burdens in clinical. Third, it could provide an optimal time window for chest $\mathrm{CT}$ review during the treatments of COVID-19 patients. This may reduce the scan times of chest CT examinations and medical cost of the patients.

Our study has several limitations. First, in this work, we focused on the principle and feasibility of the proposed method. Therefore, we did not acquire a large data sample (e.g., > 100). Second, we used visual assessment instead of the Dice coefficient to evaluate the locations of the lesions. This was due to the large differences between CT2 and follow-up CT3 images, which would lead to inaccurate image registration and Dice values [37]. In the future, registration algorithms with high performance should be developed to solve this problem.

In summary, lung ventilation maps based on two chest CT scans were used to predict the progression of lung lesions in COVID-19 patients in the early stage of the disease. The prediction maps generated from the ventilation maps could categorize the pulmonary voxels into three types according to regional function and tissue changes and then predict the potential lesions. Thus, the proposed method could help physicians predict and visualize the progression of lung lesions in COVID-19 patients, which might enable them to clearly illustrate and quickly determine an area that will be affected by pneumonia.

\section{Appendix}

\section{Calculation of $\mathrm{V}_{\text {air }}$ and $\mathrm{V}_{\text {tissue }}$ in pulmonary voxels}

A pulmonary voxel depicted in the simplified model [32] could be considered a combination of air content and tissue content. The tissue includes cells, blood, collagen, water, etc., which have approximately the same density of water. The $\mathrm{HU}$ values of air $\left(\mathrm{HU}_{\text {air }}\right)$ and tissue $\left(\mathrm{HU}_{\text {tissue }}\right)$ are -1000 and 0 , respectively. The total volume $\left(\mathrm{V}_{\text {total }}\right)$ of that pulmonary voxel is the linear combination of these two compartments

$\mathrm{V}_{\text {total }}=\mathrm{V}_{\text {air }}+\mathrm{V}_{\text {tissue }}$

In addition, the total $\mathrm{HU}$ value of that pulmonary voxel $\left(\mathrm{HU}_{\text {total }}\right)$ is considered as

$\mathrm{HU}_{\text {total }}=\left(V_{\text {air }} * H U_{\text {air }}+V_{\text {tissue }} * H U_{\text {tissue }}\right) / V_{\text {total }}$

Combining with Eq. (5) and Eq. (6), the air volume $\left(\mathrm{V}_{\text {air }}\right)$ and tissue volume $\left(\mathrm{V}_{\text {tissue }}\right)$ of that voxel could be calculated based on the total volume $\left(\mathrm{V}_{\text {total }}\right)$ and total $\mathrm{HU}$ value $\left(\mathrm{HU}_{\text {total }}\right)$ of that voxel:

$V_{\text {air }}=\frac{H U_{\text {total }}}{-1000} * V_{\text {total }}, V_{\text {tissue }}=\left(1-\frac{H U_{\text {total }}}{-1000}\right) * V_{\text {total }}$

Supplementary Information The online version contains supplementary material available at https://doi.org/10.1007/s00259-021-05435-8.

Author contribution Xin Zhou, Chaohui Ye, and Liming Xia conceived the study. Cheng Wang designed the study and proposed the method. Lu Huang collected clinical data. Cheng Wang and Sa Xiao analyzed the data. Cheng Wang and Lu Huang wrote the manuscript. Sa Xiao and Zimeng Li helped revise the manuscript. Xin Zhou and Liming Xia critically revised the manuscript.

Funding This work was supported by the National Natural Science Foundation of China (91859206, 81625011, 21921004, 82001915), the National key Research and Development Project of China (2018YFA0704000) and the Key Research Program of Frontier Sciences (ZDBS-LY-JSC004). Xin Zhou acknowledges the support from the Tencent Foundation through the XPLORER PRIZE.

Availability of data and material The datasets generated during and/ or analyzed during the current study are not publicly available due to the privacy of patients but are available from the corresponding author on reasonable request.

Code availability Code is available from the corresponding author on reasonable request.

\section{Declarations}

Ethics approval All procedures performed in this study involving human participants were in accordance with the ethical standards of the institutional and national research committee and with the 1964 Helsinki Declaration and its later amendments or comparable ethical standards. The study was approved by the ethical review board of the Innovation Academy for Precision Measurement Science and Technology of Chinese Academy of Sciences.

Consent to participate Informed consent was waived by the local ethics committee in this retrospective study.

Conflicts of interest The authors declare no competing interests.

Clinical trial registration Not applicable. 


\section{References}

1. Guan W, Ni Z, Hu Y, et al. Clinical characteristics of coronavirus disease 2019 in China. N Engl J Med. 2020;382(18):1708-20.

2. World Health Organization. Coronavirus disease (COVID-2019) situation reports. 2020. https://www.who.int/publications/m/item/ weekly-epidemiological-update---10-march-2021. Published March 9, 2021. Accessed 12 Mar 2021.

3. Fang Y, Zhang H, Xie J, et al. Sensitivity of chest CT for COVID19: comparison to RT-PCR. Radiology. 2020;296(2):E115-7.

4. Ai T, Yang Z, Hou H, et al. Correlation of chest CT and RTPCR testing for coronavirus disease 2019 (COVID-19) in China: a report of 1014 cases. Radiology. 2020;296(2):E32-40.

5. National Health Commission of the People's Republic of China. Guidelines for the diagnosis and treatment of novel coronavirus (2019-nCoV) infection (trial version 7). 2020. https://www.who. int/docs/default-source/wpro---documents/countries/china/covid19-briefing-nhc/1-clinical-protocols-for-the-diagnosis-and-treat ment-of-covid-19-v7.pdf?sfvrsn=c6cbfba4_2. Published March 3, 2020. Accessed 12 Mar 2021.

6. Wynants L, Van Calster B, Collins GS, et al. Prediction models for diagnosis and prognosis of COVID-19 infection: systematic review and critical appraisal. BMJ. 2020;369:m1328.

7. Feng Z, Yu Q, Yao S, et al. Early prediction of disease progression in COVID-19 pneumonia patients with chest CT and clinical characteristics. Nat Commun. 2020;11:4968.

8. Francone M, Iafrate F, Masci GM, et al. Chest CT score in COVID-19 patients: correlation with disease severity and shortterm prognosis. Eur Radiol. 2020;30(12):6808-17.

9. Wu G, Yang P, Xie Y, et al. Development of a clinical decision support system for severity risk prediction and triage of COVID19 patients at hospital admission: an international multicentre study. Eur Respir J. 2020;56(2):2001104.

10. Wu Q, Wang S, Li L, et al. Radiomics analysis of computed tomography helps predict poor prognostic outcome in COVID19. Theranostics. 2020;10(16):7231-44.

11. Chassagnon G, Vakalopoulou M, Battistella E, et al. AI-driven quantification, staging and outcome prediction of COVID-19 pneumonia. Med Image Anal. 2021;67:101860.

12. Chao H, Fang X, Zhang J, et al. Integrative analysis for COVID-19 patient outcome prediction. Med Image Anal. 2021;67:101844.

13. Liu F, Zhang Q, Huang C, et al. CT quantification of pneumonia lesions in early days predicts progression to severe illness in a cohort of COVID-19 patients. Theranostics. 2020;10(12):5613-22.

14. $\mathrm{Pu}$ J, Leader JK, Bandos A, et al. Automated quantification of COVID-19 severity and progression using chest CT images. Eur Radiol. 2021;31(1):436-46.

15. Chung M, Bernheim A, Mei X, et al. CT imaging features of 2019 novel coronavirus (2019-nCoV). Radiology. 2020;295(1):202-7.

16. Bernheim A, Mei X, Huang M, et al. Chest CT findings in coronavirus disease-19 (COVID-19): relationship to duration of infection. Radiology. 2020;295(3):200463.

17. Gattinoni L, Chiumello D, Rossi S. COVID-19 pneumonia: ARDS or not? Crit Care. 2020;24:154.

18. Santamarina MG, Boisier D, Contreras R, et al. COVID-19: a hypothesis regarding the ventilation-perfusion mismatch. Crit Care. 2020;24:395.

19. Colombi D, Bodini FC, Petrini M, et al. Well-aerated lung on admitting chest CT to predict adverse outcome in COVID-19 pneumonia. Radiology. 2020;296(2):E86-96.
20. King GG, Harris B, Mahadev S. V/Q SPECT: utility for investigation of pulmonary physiology. Semin Nucl Med. 2010;40(6):467-73.

21. Verger A, Bahloul A, Melki S, et al. Tracheobronchitis signs observed on ventilation lung scintigraphy during the course of COVID-19 infection. Eur J Nucl Med Mol Imaging. 2020;47:2709-10.

22. Cobes N, Guernou M, Lussato D, et al. Ventilation/perfusion SPECT/CT findings in different lung lesions associated with COVID-19: a case series. Eur J Nucl Med Mol Imaging. 2020;47:2453-60.

23. Zhou X, Graziani D, Pines A. Hyperpolarized xenon NMR and MRI signal amplification by gas extraction. Proc Natl Acad Sci USA. 2009; 106(40):16903-6.

24. Li H, Zhao X, Wang Y, et al. Damaged lung gas exchange function of discharged COVID-19 patients detected by hyperpolarized ${ }^{129}$ Xe MRI. Sci Adv. 2021;7(1):eabc8180.

25. Kipritidis J, Tahir BA, Cazoulat G, et al. The VAMPIRE challenge: a multi-institutional validation study of CT ventilation imaging. Med Phys. 2019;46(3):1198-217.

26. Guerrero T, Sanders K, Noyola-Martinez J, et al. Quantification of regional ventilation from treatment planning CT. Int J Radiat Oncol. 2005;62(3):630-4.

27. Guerrero T, Sanders K, Castillo E, et al. Dynamic ventilation imaging from four-dimensional computed tomography. Phys Med Biol. 2006;51(4):777-91.

28. Castillo R, Castillo E, Martinez J, et al. Ventilation from fourdimensional computed tomography: density versus Jacobian methods. Phys Med Biol. 2010;55(16):4661-85.

29. Fedorov A, Beichel R, Kalpathy-Cramer J, et al. 3D Slicer as an image computing platform for the quantitative imaging network. Magn Reson Imaging. 2012;30(9):1323-41.

30. Klein S, Staring M, Murphy K, et al. Elastix: a toolbox for intensity-based medical image registration. IEEE T Med Imaging. 2009;29(1):196-205.

31. Castillo R, Castillo E, Mccurdy M, et al. Spatial correspondence of 4D CT ventilation and SPECT pulmonary perfusion defects in patients with malignant airway stenosis. Phys Med Biol. 2012;57(7):1855-71.

32. Simon BA. Non-invasive imaging of regional lung function using X-ray computed tomography. J Clin Monit Comput. 2000;16(5):433-42.

33. Kipritidis J, Siva S, Hofman MS, et al. Validating and improving $\mathrm{CT}$ ventilation imaging by correlating with ventilation 4D-PET/CT using ${ }^{68} \mathrm{Ga}$-labeled nanoparticles. Med Phys. 2014;41(1):011910.

34. Yamamoto T, Kabus S, Lorenz C, et al. Pulmonary ventilation imaging based on 4-dimensional computed tomography: comparison with pulmonary function tests and SPECT ventilation images. Int J Radiation Oncol Biol Phys. 2014;90(2):414-22.

35. He M, Zha W, Tan F, et al. A comparison of two hyperpolarized ${ }^{129} \mathrm{Xe}$ MRI ventilation quantification pipelines: the effect of signal to noise ratio. Acad Radiol. 2019;26(7):949-59.

36. Pan F, Ye T, Sun P, et al. Time course of lung changes at chest CT during recovery from coronavirus disease 2019 (COVID-19). Radiology. 2020;295(3):715-21.

37. Cohade C, Osman M, Marshall LT, et al. PET-CT: accuracy of PET and CT spatial registration of lung lesions. Eur J Nucl Med Mol Imaging. 2003;30:721-6.

Publisher's note Springer Nature remains neutral with regard to jurisdictional claims in published maps and institutional affiliations. 